\title{
Monitoring Collaboration from a Value Perspective
}

\author{
Lianne Bodenstaff*, Andreas Wombacher ${ }^{\dagger}$, Manfred Reichert* and Roel Wieringa* \\ *Information Systems Group, Department of Computer Science, \\ University of Twente, The Netherlands \\ Email: \{1.bodenstaff,m.u.reichert,r.wieringa\}@utwente.nl \\ ${ }^{\dagger}$ School of Computer and Communication Sciences, \\ École Polytechnique Fédérale de Lausanne (EPFL), Switzerland \\ Email: andreas.wombacher@epfl.ch
}

\begin{abstract}
Collaborations among businesses can be described from different viewpoints. Two of these viewpoints are the value viewpoint, representing estimated values exchanged in a collaboration, and the coordination viewpoint, representing messages exchanged between the actors to coordinate the execution of a collaboration. To observe and maintain the value viewpoint during the complete life cycle, the estimated values have to be validated during the execution of the collaboration. However, since the value model is not implemented, the necessary information for monitoring the value viewpoint needs to be derived from the coordination viewpoint. Relating coordination and value viewpoint is a difficult process because the coordination viewpoint lacks information present in the value viewpoint. In this paper we define the relation between both viewpoints for the complete collaboration life cycle. Furthermore, we provide a mechanism to monitor the collaboration from a value viewpoint.
\end{abstract}

\section{INTRODUCTION}

Monitoring the business from a value viewpoint is important for decision making on the profitability of a cooperation during the lifetime of this cooperation. A value model gives an indication on the profitability of an inter-organizational business cooperation. It describes the value viewpoint of the cooperation by estimating the number of objects with economic value exchanged between the business partners. Business decisions are based on the estimated economic behavior of the different parties in the cooperation. The coordination model describes how this cooperation can be realized. However, the value viewpoint should not only be considered during the process of business decision making. It should be considered during the entire life cycle of the cooperation, monitoring the profitability during runtime. In this paper we answer the question on how to monitor the collaboration from a value perspective.

Since the value model is not directly implemented, a collaboration is executed by implementing the coordination model. Therefore, monitoring is based on the coordination model. Monitoring is enabled by explicating the relation between coordination model and value model. Information present in the log files of the implemented coordination model has to be related to exchanges in the value model. However, the relation between these two models is not straightforward since the

This research has been supported by the Netherlands Organisational for Scientific Research (NWO) under contract number 612.063.409 coordination model lacks information necessary for evaluating the value perspective. For example, the coordination model contains information about the messages exchanged between the parties but provides no information about the exchanged objects of economic value in that specific message. Therefore, the value perspective is of importance for monitoring the economic results of the cooperation during runtime. Through monitoring it is checked whether the results are consistent with the original estimations made in the value model.

To monitor the value perspective of the cooperation during runtime, the relation between the value model and coordination model has to be established. In this paper we relate both models by checking consistency. We give static and dynamic consistency definitions, where static consistency relates both models during design time and dynamic consistency relates both models during runtime. Furthermore, we introduce an approach to achieve dynamic consistency through actively adapting the value model according to the results provided by the coordination model. With this approach we provide a mechanism to monitor the business from a value perspective.

This paper is structured as follows: in Section II our running example used for illustrating our approach is introduced. Furthermore, the business case is represented as a value model and part of the business case is represented as a coordination model. Section III introduces the concepts of static and dynamic consistency. Our approach in relating value and coordination model through consistency is discussed in Section IV. Section V discusses related work. We conclude this paper with a summary and outlook in Section VI.

\section{Modelling the Business CAse}

In this paper we use a running example based on a real case in the health insurance sector in the Netherlands for illustrating our approach. An insurance company provides insurance on an annual basis to its customers. The customers pay premium on a monthly basis and can claim refunds for received treatments. Every paid refund by the insurance company is compensated by CVZ, a Dutch organization distributing tax money. CVZ gets its funding from the government. Next, we model this scenario as a value model and part of the scenario as a coordination model introducing the required concepts. 


\section{A. Value Model}

For evaluating the economic profitability of a cooperation a value model is created. The value model of our business case is defined using $\mathrm{e}^{3}$-value [1](cf. Figure 1). In $\mathrm{e}^{3}$-value, Net Present Value (NPV) [2] is used for cost-benefit analysis of the cooperation to evaluate the revenue for every actor. We chose $\mathrm{e}^{3}$-value because of its graphical representation. The approach described in this paper is, however, applicable to value models in general.

Our business case represented as $\mathrm{e}^{3}$-value model is described in detail in a technical report [3]. In this paper we restrict ourselves to those constructs necessary for understanding the approach.

The example from Figure 1 comprises four actors and eight value transfers. For example, the value object premium is transferred from the customer to the insurance company. Another value object, the insurance itself, is transferred from the insurance company to the customer. In Figure 1 these two transfers are annotated with an 'F'. A combination of value transfers in one transaction is referred to as a value exchange. In $\mathrm{e}^{3}$-value a distinction is made between different kinds of value objects. A value object either is a product, service, money or consumer experience. In this example the premium is a value object of type money and the insurance provided by the insurance company can be considered as a service.

The consumer need is "having a health insurance for one year". This is represented by placing the start stimulus at the customer. The set of value objects that has to be transferred to fulfill the consumer need, consists of all value transfers connected through the dependency path in the model. Every month there are two possible sets of value transfers that can fulfill the consumer need. Either the customer claims restitution for treatments he paid for himself and he pays the monthly premium, or he only pays the monthly premium. When the customer claims a restitution, the insurance company claims compensation from CVZ. CVZ, in turn, gets its funding from the government. Also note that the health insurance company has multiple customers, represented as a market segment in Figure 1.

In Figure 1, the twelve monthly payments for fulfilling one consumer need are realized by adding an explosion element, annotated with ' $\mathrm{A}$ ' in the figure, associated with ratio $1: 12$. The choice between the two options for fulfilling the consumer need is represented as an OR-split. After such an OR-split only one of the dependency paths is selected. When the customer has not received treatments that month, the path annotated with ' $C$ ' is chosen. The two resulting value transfers constitute the first set of transfers that can fulfill the consumer need. Otherwise, if the customer received treatment that month, the path annotated with ' $D$ ' is chosen. This path further splits through an AND-split, representing a parallel occurrence of two or more dependency paths. In an AND-join, in turn, all incoming dependency paths share the continuation of the dependency path. The two value exchanges between the customer and the insurance company take place. To enable

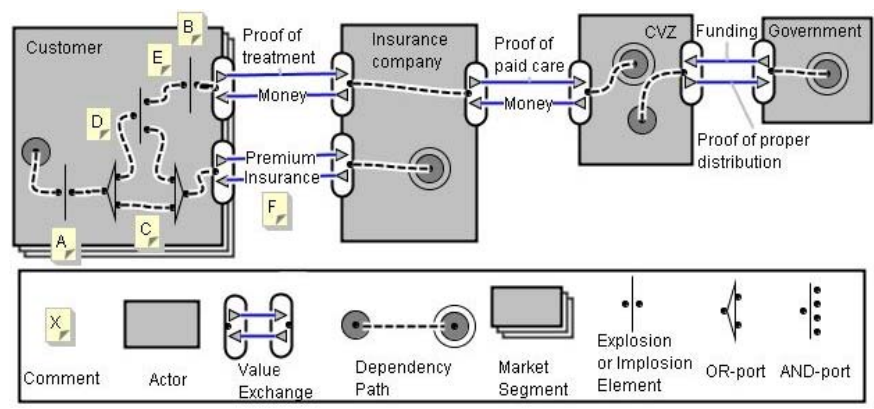

Fig. 1. $\mathrm{e}^{3}$-value model, the business case

more than one restitution claim per month another explosion element, annotated with ' $\mathrm{B}$ ', is added. The insurance company claims restitution from CVZ. These value transfers constitutes the second set of value transfers. Finally, the dependency path starting within CVZ represents the third set of value transfers.

In the profitability sheets (not shown in Figure 1), which can be associated with the graphical representation of the value model, the estimations are given. The market segment, for example, can be quantified by estimating the number of customers and the ratio on the explosion elements and ORsplit is set. For every monetary value transfer a quantification is given in the profitability sheets. The expected revenue for every actor in the model can be calculated.

\section{B. Coordination Model}

A coordination model depicts how the transfer of value objects between the parties is realized. In particular, it describes the order in which messages between parties are exchanged. An ordered set of messages is referred to as execution sequence. This ordering information is omitted in the value model. Examples of formalisms for defining coordination models are Petri Nets and Activity Diagrams.

Due to lack of space we depict a small example of a coordination model represented as a Petri Net in Figure 2 instead of a graphical representation of our business case. However, [4] provides a detailed description of the business case as well as the model represented as a Petri Net [5]. Figure 2 represents the coordination of the payment process of a customer to the insurance company for having insurance for one year. This part of the coordination process is related to the value exchange of premium and insurance in the value model, annotated with ' $F$ ' in Figure 1. The money value transfer premium is represented as a message exchange in the coordination model. The service value transfer insurance is not, since services do not instantiate explicit message exchanges.

Places (indicated as circles) can hold any number of tokens (represented as black dots) and transitions (indicated as squares) act on input tokens by firing. Message exchanges are represented as places and tasks as transitions in the coordination model. Places and transitions are connected through arcs. These arcs indicate the ordering of tasks and message exchanges. In Figure 2 the customer has first to make a payment through executing task Pay after which message 


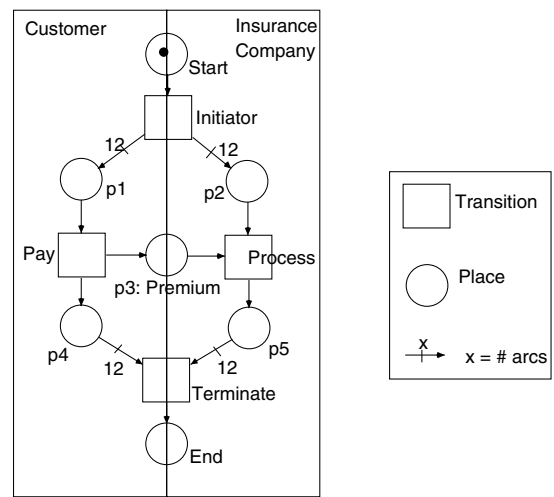

Fig. 2. Petri Net, example

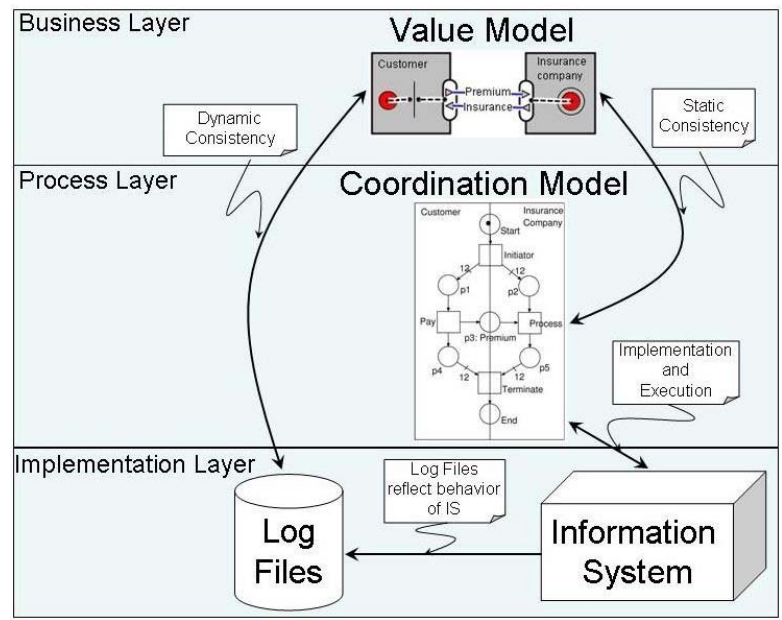

Fig. 3. Consistency relations

Premium, place p3 in Figure 2, is transferred from customer to insurance company.

\section{STATiC AND DyNAmiC CONSISTENCY}

To relate value and coordination model we define a consistency relation which ensures that both models describe the same system. We consider two types, namely static and dynamic consistency. Static consistency refers to checking static model aspects without considering the model behavior during runtime. By contrast, dynamic consistency takes the dynamic model behavior into account for two static consistent models. Figure 3 depicts the relations between the different systems. Dynamic consistency relates value and coordination model during runtime through data gathered in the log files. These log files reflect the behavior of the Information System. In turn, the Information System implements and executes the coordination model. Other work like e.g. [6] focusses on directly relating log file and coordination model.

For defining static consistency we rely on the intuitive definition of consistency between value and coordination models given in [7].
Definition $1 A$ value and coordination model are considered to be statically consistent if:

(i) for every set of value transfers in the value model (dependency path in $e^{3}$-value), there exists an execution sequence in the coordination model such that exactly the product/money value transfers contained in the set are exchanged in the execution sequence, and

(ii) for every execution sequence in the coordination model, there exists a set of value transfers in the value model (dependency path in $e^{3}$-value) such that the message exchanges contained in the execution sequence represent product/money value transfers exchanged in the set of value transfers.

Next we consider dynamic consistency. In this paper we assume a strict relation between coordination model and log files which is defined as follows.

Definition 2 A coordination model and log file have a strict relation if:

(i) and only if for each message exchange in the coordination model there is an entry in the log file, and

(ii) the order of message exchanges in the coordination model is equal to the order of entries in the log file.

Furthermore, two aspects have to be considered for our dynamic consistency definition. First, the total value of the transferred messages should be equal to the estimations made in the value model. If, for example, the average value of received orders is lower than the estimated average value of each order then the two models are considered as being not consistent with each other. Second, the number of occurred value transfers has to be equal to the estimated amount of occurrences. If, for example, the estimated number of orders is twice as high as the realized number of orders then the models would not be considered consistent. Taking these two aspects into account, we define $\delta$ dynamic consistency as follows.

Definition 3 A value and a coordination model are considered to be $\delta$ dynamically consistent at time $t$ if for all message exchanges $x$ in the log file representing value transfers $y$ in the value model it holds that:

(i) the average number of realized $x$ between time $t-\delta$ and $t(\delta>0)$ is equal to the number of estimated occurrences of $y$, and

(ii) the average value of $x$ between time $t-\delta$ and $t(\delta>0)$ is equal to the estimated value of $y$.

In this context, dynamic consistency checking is based on calculating averages over a period of time $\delta$. The two models are considered to be consistent with each other if the average value and the average number of occurrences were consistent during that period of time. If necessary, constraints of this definition can be relaxed dependent on the nature of the business and the value objects that are transferred. 


\section{APPROACH}

$\delta$ dynamic consistency forms the basis for our approach on relating value and coordination model. We use the data from the log file as feedback information for the value model. The value model is actively adapted during runtime using the data perceived from the monitoring process. This approach provides a mechanism for monitoring the business from a value perspective.

Item (ii) of Definition 3) addresses the average value of each transfer. The average value of each transfer is represented in the log files. The monitored value of the transfers is compared with the estimations made in the value model. This is described in Subsection IV-A.

Subsections IV-B, IV-C, IV-D concern item (i) of Definition $3)$. The $\log$ files contain the information about the messages exchanged between the different actors. These messages, in turn, hold the information on the number of value transfers that occurred between the actors. For using this information in evaluating the value model, a correlation between both models and their constructs has to be established. When constructing the value model several estimations are made regarding the behavior of the system like e.g. consumer needs and ratios on explosion elements. The estimated number of value transfer occurrences is based on these estimations. An equation system can be derived which comprises the formulas for calculating the number of occurrences based on the used constructs in the value model. Data from the $\log$ files is entered into the equation system. When solving the equation system with these values there may be free variables representing the ratios. These free variables are represented in a Graphical User Interface.

After the adaptation of the ratios in the $\mathrm{e}^{3}$-value model, these results are graphically represented together with the result of monitoring the value of the transfers. This is demonstrated in Subsection IV-E. In the following sections, the different parts of the approach are explained in detail and illustrated on behalf of our example business case. Due to lack of space, the approach is explained only on behalf of the customer which is sufficiently complex.

\section{A. Average Value of Transfers}

The value of the transfers is monitored in the log files. Figure 4 represents the estimations made on the value of each transfer as well as the monitored average of each value transfer. We monitor the coordination process for the average value of each transfer by calculating the moving average. Since we assume in this example that the customer has constant behavior over time, we are able to use time series. Every quarter the average value of a transfer over the preceding year is calculated. Using a moving average results in a smoothly changing average over the time series. Figure 4 depicts measurements in quarter Q4 of 2005, quarter Q1 of 2006 and quarter Q2 of 2006, annotated with T1, T2 and T3, respectively. For calculating the realized average value of a transfer over a year, each quarter average should be multiplied by the number of realized transfers and the total should be

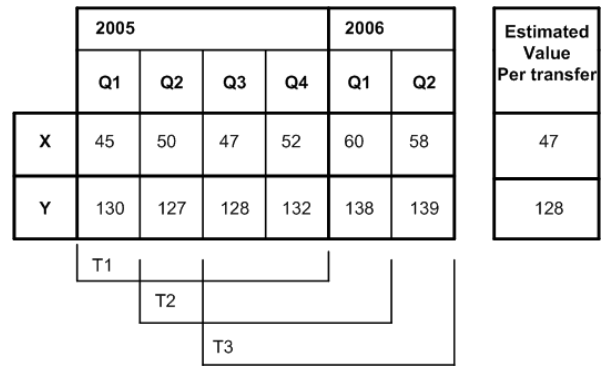

Fig. 4. Average Value of Transfers

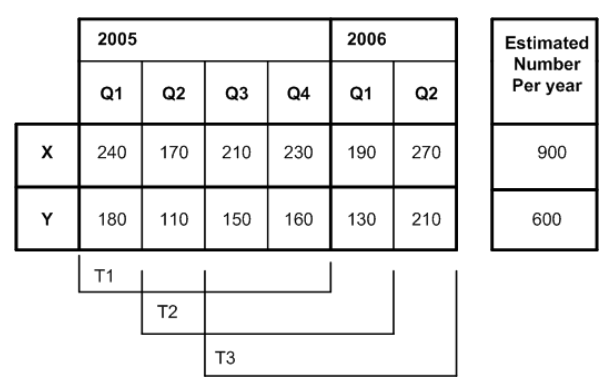

Fig. 5. Average Number of Transfers

divided by the total number of realized transfers over that year.

\section{B. Average Number of Transfers}

During the execution of the coordination model, we monitor the coordination process by calculating the moving average as depicted in Figure 5. In the figure the number of message transfers as observed at different times during execution are depicted. In our example every quarter the averages over the preceding year are calculated. Figure 5 depicts measuring in quarter Q4 of 2005, quarter Q1 of 2006 and quarter Q2 of 2006, annotated with T1, T2 and T3, respectively. These message exchanges are correlated to the value transfers in the value model. This correlation is based on static consistency as specified in Definition 1. ${ }^{1}$

\section{The Equation System}

The equation system enables calculating the number of occurrences through formulas based on the constructs of the value model. The equation system of a value model is constructed through use of an algorithm. The value model is represented as a graph where vertices represent constructs and edges represent parts of dependency paths. The equation system is constructed by assigning variables to each part of the dependency path between the different constructs of the value model. The equations in the system can be related to each other through these variables. When two constructs are directly connected with each other through a dependency path, the variable used in the equations of both constructs will be the same. The algorithm is as follows:

\footnotetext{
${ }^{1}$ for details see [3]
} 


\begin{tabular}{|ll|ll|}
\hline $\begin{array}{l}\text { OR-split: } \\
y=\frac{r}{r+s} x\end{array}$ & $s_{r}$ & $\begin{array}{l}\text { Start: } \\
z=\frac{s}{r+s} x\end{array}$ &
\end{tabular}

TABLE I

EQUATION SYSTEM

For each edge assign a unique variable. For each vertex determine the edges and associated variables. Represent each vertex as an equation based on the equation system in Table I with instantiating the variables as the assigned values.

For each construct of the value model a formula exists in Table I. For example, in Figure 1 there is one dependency path which enters an OR-split whereas two paths leave this element. For the fulfillment of a consumer need, only one of the outgoing paths is chosen. In the profitability sheets an estimation is made on the relation between the number of times each path is chosen. In the table this is indicated by $r$ and $s$, stating that path $y$ is chosen $r$ times and path $z$ is chosen $s$ times. The resulting formula states that the estimated number of times path $y$ is chosen is equal to the number of times dependency path $x$ occurs times the ratio on the OR-split, namely $\frac{r}{r+s}$. In $\mathrm{e}^{3}$-value an OR-port is an exclusive OR. For the OR-join the number of occurrences of both incoming paths can be added up. For the AND-join as well as for the ANDsplit the number of occurrences on each part of the dependency path is the same. An implosion or explosion element multiplies the number of occurrences with the ratio $\frac{s}{r}$ associated with the port. The number of occurrences on outgoing path $x$ of a startstimulus equals the number of occurences of consumer need $y$ times the number of actors $z$ in the market segment. The number of occurrences on stop-stimulus $y$ equals the number of occurrences of incoming dependency path $x$ times the number of actors $z$ in the market segment. When the market segment consists of a single actor the value of $z$ equals 1 . The formula Transfer of Table I states that the outgoing number of value transfers $y$ is equal to the number of occurrences $x$ times ratio $s$ and that the incoming number of value transfers $z$ is equal to the number of occurrences $x$ times ratio $t$. This ratio represents for example several payments for receiving one value object.

We illustrate our approach by deriving the equation system for the customer. Figure 6 depicts the customer with the ratios and introduced variables as they are used for the equation

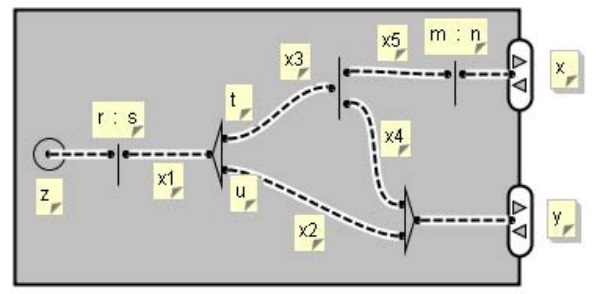

Fig. 6. Customer, ratios and introduced variables

\begin{tabular}{|c||c||c||c||c|}
\hline & $\begin{array}{c}\text { Estimated } \\
\text { Numbers }\end{array}$ & T1 & T2 & T3 \\
\hline$x$ & 900 & 850 & 800 & 900 \\
\hline$y$ & 600 & 600 & 550 & 650 \\
\hline$z$ & 50 & $\mathrm{z}$ & $\mathrm{z}$ & $\mathrm{Z}$ \\
\hline$f_{1}$ & 12 & $\frac{600}{z}$ & $\frac{550}{z}$ & $\frac{650}{z}$ \\
\hline$f_{2}$ & $\frac{3}{4}$ & $\frac{17}{12 f_{3}}$ & $\frac{16}{11 f_{3}}$ & $\frac{18}{13 f_{3}}$ \\
\hline$f_{3}$ & 2 & $f_{3}$ & $f_{3}$ & $f_{3}$ \\
\hline
\end{tabular}

TABLE II

LOG FILES

system. Since this example is for demonstration purposes only, we consider the customer to be a single actor and we assume there is no ratio on the value interfaces. Next, the resulting equation system, without pure renaming, is represented. For a synoptic representation of the graphical user interface, the ratio on the first explosion element, $\frac{s}{r}$, is referred to as fraction $f_{1}$ and the ratios on the OR-split, $\frac{u}{t+u}$ and $\frac{t}{t+u}$, are referred to as fractions $1-f_{2}$ and $f_{2}$, respectively. The ratio on the second explosion element, $\frac{n}{m}$ is referred to as fraction $f_{3}$.

- $x_{1}=f_{1} z$ with $f_{1}=\frac{s}{r}$

- $x_{2}=\left(1-f_{2}\right) x_{1}$ with $1-f_{2}=\frac{u}{t+u}$

- $x_{3}=f_{2} x_{1}$ with $f_{2}=\frac{t}{t+u}$

- $x_{5}=x_{3}$

- $x_{4}=x_{3}$

- $y=x_{4}+x_{2}$

- $x=f_{3} x_{5}$ with $f_{3}=\frac{n}{m}$

\section{Adapting the Equation System}

In the value model estimations are made for the number of consumer needs and the different ratios of the constructs. Based on these estimations the estimated number of value transfers can be calculated. In Table II the entries in Estimated Numbers column depict these estimations and calculations. The information from the log files is correlated with the value transfers and the results at three different times, T1, T2, T3, depicted in Table II. The observed average of message exchanges during monitoring can deviate from the estimations made in the value model. For example, the measurements on the number of value transfers $x$ at time T1, namely 850 , deviate from the estimated number of value transfers $x$ in the value model, namely 900 . This result makes the value model and coordination model $\delta$ dynamically inconsistent according to Definition 3. 


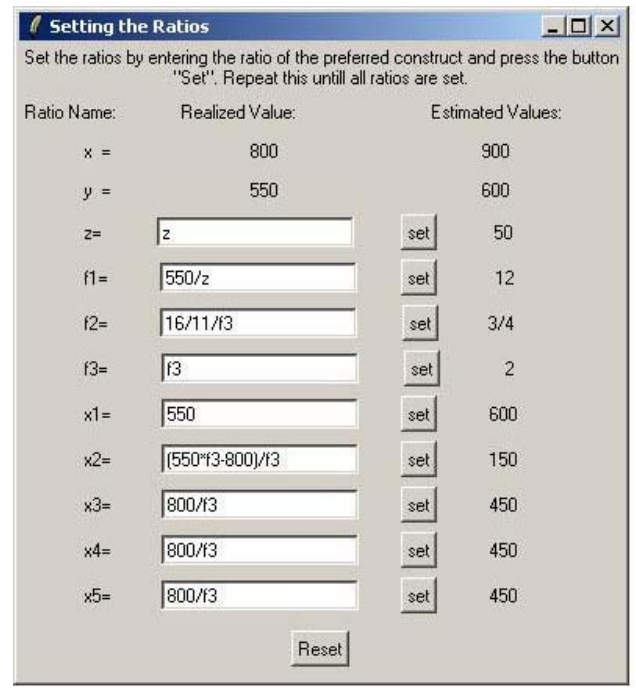

Fig. 7. The Graphical User Interface

When the value model is not $\delta$ dynamically consistent with the coordination model one or more of the estimated ratios in the value model have to be adapted to make the models $\delta$ dynamically consistent. Entering the results of the log files into the equation system as derived from the value model while keeping the ratios as free variables and solve the equation system can have two types of solutions. In the first case the equation system is over specified and there is no or just one solution to the equation system, in which case there is no necessity for a graphical user interface. In the second case the equation system is under specified leaving a possible infinite number of solutions. In the latter case, representing the options in a graphical user interface allows the user to dynamically set the different ratios and visualize the effects on the value model. The visualization is important for evaluating the economical value of the business during runtime.

An example of this graphical user interface is depicted in Figure 7. Here, the results of the log files from executing the coordination model at time $\mathrm{T} 2$ are depicted. When the user chooses a free variable to set and enters this value into the user interface then the equation system is reevaluated. The results, possibly still with free variables, are again represented in the graphical user interface. After setting all free variables, the ratios are adapted in such a way that the value and coordination model are one year dynamically consistent.

\section{E. Visualization of the Results}

In this paper only one actor in a simplified business case is evaluated. Real life business constellations, however, are more complex and potentially consist of many constructs. Adapting one of the ratios in the value model can influence many other ratios and estimations in the value model. These effects are visualized in the value model by adapting the colors of the constructs automatically according to the adaptation of the ratios. The user can directly see what the effects are of adapting one specific ratio on the entire business constellation.
Here, the effects of the entered values on the value model, compared with the original estimated values of the value model are calculated, classified and represented by an appropriate color. In the example a construct is colored green when the entered or calculated ratio matches the estimations made in the value model. A construct is colored dark green when the ratio deviates less than $8.5 \%$ from the estimated value and it is colored red when the ratio deviates over $8.5 \%$ from the estimations made in the value model.

In Figure 8 examples of the value model coloring for time series T2 from Figure 5 and Figure 4 are given. Figure 8 (a) shows the value model after entering the results of T2. The average value of transfer $x$, a restitution, is in timeseries T2 52.16 (cf. Figure 4). This is more than $8.5 \%$ deviation of the estimated value 47 . Therefore, the lines of value transfer $x$ are colored red. The average value of transfer $y$, the premium, is in timeseries T2 131.33. This is less than $8.5 \%$ deviation and therefore the lines of value transfer $y$ are colored dark green. The average number of occurrences of $x$ in T2 is 800 while the estimation in the value model for $x$ was 900 (cf. Figure 5). The deviation between the realized number of value transfers $x$ and the estimated amount is greater than $8.5 \%$ and therefore the interface is colored red. The realized number of $y$ is 550 while the estimated number was 600 . The deviation is less than $8.5 \%$, therefore the interface is colored dark green. The remaining variables are still open and therefore grey.

Figure 8 (b) depicts the situation after setting the ratio for f1. f1 denotes the number of payments each customer makes for having insurance for one year. Since each customer pays every month its premium, this is a fixed ratio of 12 . When choosing which ratios to adapt in the value model, f1 will not be chosen since it is a fixed ratio. Therefore, this is the first ratio to be set in the graphical user interface. After entering the value 12 for variable $\mathrm{f} 1$, the explosion element on which ratio f1 is set, changes into the color green. This indicates that the estimated ratio of $1: 12$ is equal to the realized ratio. As a consequence, the realized number of consumer needs, $z$, becomes 45.8. The estimated number of consumer needs was 50. The deviation is within $8.5 \%$. Therefore, the start stimulus is colored dark green.

In Figure 8 (b) a possible final marking is depicted. When we assume there is additional information available on the percentage of customers that use the possibility of asking restitution in a month then the ratio on $\mathrm{f} 2$ can be set. We assume this ratio is $70 \%$. The deviation between the estimated ratio of $75 \%$ and the realized ratio of $70 \%$ is lower than $8.5 \%$. Therefore, the OR-port associated with $\mathrm{f} 2$ is colored dark green. As a result, f3 is assigned a value of approximately 2.1 which is also within the $8.5 \%$ deviation. The explosion element associated with ratio $\mathrm{f} 3$ is colored dark green.

The conclusion from these results is that there have been less people asking for a restitution on a monthly basis but if a customer asked for a restitution, they asked for more restitutions than estimated. This is indicated by the slightly increased ratio on $\mathrm{f} 3$. 


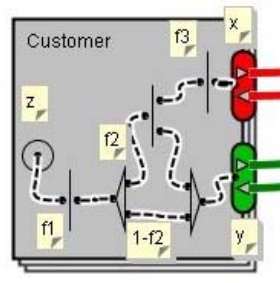

(a)

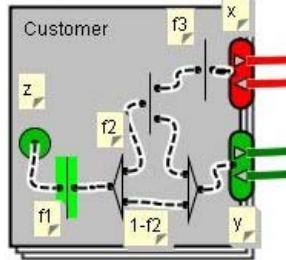

(b)

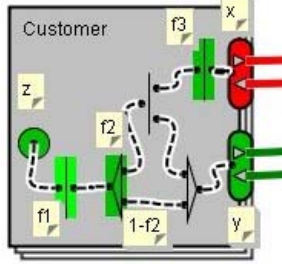

(c)
Fig. 8. Coloring of the Value Model

\section{RELATED WORK}

Consistency between different viewpoints has been addressed on different levels of abstraction. An analysis on the conceptual level has been provided in [8] where the value and coordination viewpoints are compared based on the semantic concepts used in the different viewpoints have. However, this approach does not provide a means to compare two concrete models. A human intuitive consistency definition has been proposed in [9] which gives an understanding on what consistency means without explaining how to check it.

A well known approach for assessing business models is using Key Performance Indicators (KPI). In these approaches, KPI are chosen as evaluation criteria for business models. In [10] KPI are used to overcome the problem of measuring a priori the benefits of e-commerce investments. The e-business is assessed by business process simulation where users can experiment with different configurations. The resulting simulated values of the KPI are compared with the estimated values in the process models. A business decision is made based on this comparison. In our mechanism, the profitability evaluation criterium can be considered a KPI.

Another approach is forecasting modelling where a prediction on future behavior is made based on current available data. These models are used for decision making. In [11] decisions on cooperative investments are supported using forecasting models. Here, also simulation models are used for selecting proper forecasting models. However, these approaches do not provide a business view on the dependencies of the measured values. Furthermore, these approaches do estimations on the future rather than representing observed behavior.

Another mechanism for adapting models during runtime is the use of reflection. [12], for example, separates representation and enactment domains which relates to our separation of design time and runtime environments. Their approach supports ongoing transformations between both domains. The use of reflection allows generation of new programs by another running program. This allows users to add new processes to a running program. In [13] reflection is used for reusing, extending and customizing current processes. While these approaches using reflection focus on evolution of processes, they disregard monitoring the business from a value viewpoint.

\section{CONCLUSION AND OUtLOOK}

In this paper we propose a mechanism on monitoring business from a value perspective. We relate the value model and coordination model through consistency relations. The contribution of this paper is the definition of $\delta$ dynamic consistency which enables correlation of value models and coordination models. Furthermore, we introduced an approach to enable monitoring the value model based on results of the cooperation during runtime. The mechanism supports decision making on the profitability of the cooperation as well as adaptation of the value model during runtime.

We continue our work by considering complex situations as noisy logs, error handling and season dependent customer behavior. Furthermore, the visualization of the results of adapting the value model will be extended, using a more extensive coloring of the constructs in the value model. In this paper the focus was on adapting the value model, in our future work we will investigate the possibility of feedback relations from the adapted value model to the coordination model. Possibly resulting in an interactive environment where both models are dynamically adapted due to the consistency relations.

\section{REFERENCES}

[1] J. Gordijn and J. M. Akkermans, "Value-based requirements engineering: Exploring innovative e-commerce ideas." Requirements Engineering, vol. 8, no. 2, pp. 114-134, 2003.

[2] K. Laudon and J. Laudon, Essentials of Management Information Systems, 5th ed. Prentice Hall, 2003.

[3] L. Bodenstaff, A. Wombacher, and M. Reichert, "Dynamic consistency between value and coordination models - research issues," CTIT: Centre for Telematics and Information Technology, P.O. Box 217 - 7500 AE Enschede - The Netherlands, Technical Report 06-50, 2006.

[4] - "Dynamic consistency between value and coordination models research issues," 2006.

[5] K. Jensen, Coloured Petri Nets. Basic Concepts, Analysis Methods and Practical Use. Springer, 1997, three Volumes.

[6] W. M. P. van der Aalst, B. F. van Dongen, J. Herbst, L. Maruster, G. Schimm, and A. J. M. M. Weijters, "Workflow mining: A survey of issues and approaches," Data \& Knowledge Engineering, vol. 47, no. 2, pp. 237-267, 2003.

[7] Z. Zlatev and A. Wombacher, "Consistency between $\mathrm{e}^{3}$-value models and activity diagrams in a multi-perspective development method," in OTM Conferences (1), 2005, pp. 520-538.

[8] J. Gordijn, J. Akkermans, and J. van Vliet, "Business modelling is not process modelling," in Conceptual Modeling for E-Business and the Web, vol. 1921. Springer LNCS, 2000, pp. 40-51.

[9] R. J. Wieringa and J. Gordijn, "Value-oriented design of service coordination processes: correctness and trust," in Proceedings of the ACM Symposium on Applied Computing (SAC. New York, NY, USA: ACM Press, 2005, pp. 1320-1327.

[10] G. Giaglis, R. Paul, and G. Doukidis, "Dynamic modelling to assess the business value of electronic commerce," in Bled: Proceedings of the 11th International Electronic Commerce Conference, 1998.

[11] X. Zhao, J. Xie, and J. Leung, "The impact of forecasting model selection on the value of information sharing in a supply chain," European Journal of Operational Research, vol. 142, pp. 321-344, 2002.

[12] R. M. Greenwood, D. Balasubramaniam, G. N. C. Kirby, K. Mayes, R. Morrison, W. Seet, B. Warboys, and E. Zirintsis, "Reflection and reification in process system evolution: Experience and opportunity," in EWSPT '01: Proceedings of the 8th European Workshop on Software Process Technology. London, UK: Springer-Verlag, 2001, pp. 27-38.

[13] D. Edmond and M. Papazoglou, "Reflection is the essence of cooperation," in Cooperative Information Systems: Current Trends and Directions, M. P. Papazoglou and G. Schlageter, Eds. Academic Press, 1998, pp. 233-262. 